\title{
Zinc Sulfide Waveguide Analysis
}

\author{
Abd-Alrahman Khalid", Nabeil I. Fawaz \\ Department of Physics, College of Science, University Of Anbar, Iraq
}

Received March 16, 2020; Revised May 23, 2020; Accepted June 4, 2020

Copyright $\subseteq 2020$ by authors, all rights reserved. Authors agree that this article remains permanently open access under the terms of the Creative Commons Attribution License 4.0 International License

\begin{abstract}
The Maxwell equations is considered as a fundamental formulation of wave propagation for a dielectric slab waveguide at frequencies in the IR region. In this article, the theoretical analysis of electromagnetic waves propagating through the zinc sulfide ( $\mathrm{ZnS}$ ) semiconductor planar waveguide at wavelength of $1 \mu \mathrm{m}$ is reported using 2013 MATLAB software. Additionally, a comparison of the waveguide parameters using two modes namely transvers electric (TE) and transvers magnetic (TM) is demonstrated in details. This could be attributed to the interference of effective refractive index value. In particular, the penetration depth has demonstrated values of 0.2419 and $0.2445 \mu \mathrm{m}$ for $\mathrm{TE}_{0}$ and $\mathrm{TM}_{0}$ modes at thickness of $2.69 \mu \mathrm{m}$, respectively. Subsequently, the effect of temperature on the effective reflective index has been elaborated. Furthermore, the propagation angles in TM modes exhibited lower values than TE modes with respect to the core thickness; and lower wavelength resulted in higher cutoff frequency with respect to the number of modes. While, the relation between $n_{\text {eff }}$ and temperature for both TE and TM modes showed that TE mode exhibited advanced values as compared to TM mode. This work demonstrates a short pathway for a theoretical analysis of electromagnetic waves through $\mathrm{ZnS}$ planer waveguide using both TE and TM modes.
\end{abstract}

Keywords Planar Waveguide, Zinc Sulfide, Transvers Electric, Transvers Magnetic

\section{Introduction}

The electromagnetic waves propagation behavior of a dielectric material is essential prospect in which the energy is transferred from one point to another. An effective energy transfer between two points in a specific pathway is carried out using a particularly designed electromagnetic geometry. These geometries are known as waveguides in which minimal energy loss is acquired. These waveguides are generally divided into two main and very distinct classifications namely metallic and dielectric waveguides; which are used in binary detached electromagnetic wave regimes. The dielectric waveguide, which is also known as a planar waveguide, is the simplest form of a waveguide structure. This could be due to the easily described guided modes of waveguides geometry [1-3]. The bases of waveguides in a dielectric waveguide are based on the electromagnetic theory of light and its interaction with matter. These bases have been well-known for more than a century and one of its common applications is known as optical devices. An optical device is a structure of simple waveguide and it is consisted of three different layers which are substrate, core and cladding [4]. The waveguide modes are described on the bases of their propagation constant; which is a speed measure by which phase fronts are propagated throughout the structure [5].

Electromagnetic wave, in a waveguide, is propagated in a separate set of states in which these states are commonly known as modes. Modes, in general, are mathematical representation used to describe the nature of electromagnetic wave propagation in a waveguide [6]. A transverse mode of electromagnetic wave is defined as a specific electromagnetic radiation pattern through a plane which is perpendicular to the direction of radiation propagation. Transverse modes occurring in micro and radio waves are limited to waveguides [7]. These modes are classified in two categories namely TE and TM. TE mode is found with no electric field in the propagation direction. A TE mode is also distinct by the symbol $\mathrm{H}$ due to the occurrence of the magnetic field along the propagation direction. In the meanwhile, TM is sometimes defined by the symbol $\mathrm{E}$ as the electric field is the only field in which is propagated along the wave direction with no presence of magnetic field [8].

In this study, the substrate and cladding are operated with the same refractive index so the waveguide is then called a symmetric waveguide. A waveguide doing as a transducer that transform perturbations such as temperature, strain, voltage, rotation or electric current and magnetic changes to be convenient to radiation [4]. This research focuses on studying wave transmission within the 
waveguide and clarifying graphical relationships between parameters in TE and TM modes.

\section{Theory}

\subsection{Dielectric Planar Waveguide}

It is generally known that a dielectric slab waveguide, as demonstrated in Figure 1, is a planar dielectric thin film or also known as planar dielectric sheet which has thickness of $2 \mathrm{a}$ [9].

Herein, the wave propagation in the direction of $\mathrm{z}$ is occur due to the total internal reflection of the slab from its right as well as left internal walls. In optical fibers, this type of waveguides delivers simple and easy processed models for the wave propagation confining mechanism. Fields which are propagated undergo the confined mechanism mainly inside the slab, but these fields exist in the form of waves outside the slab. This leads to exponential decay with the separating distance to the slab. Figure 1 illustrates characteristic pattern of the electric field as a function of $\mathrm{x}$. For uncomplicatedness, it is assumed that the slab media to the right and left are symmetric. In order to guarantee complete internal reflection, the dielectric constants outside and inside the slab ought to fulfill the condition of $\mathrm{n} 1>\mathrm{n} 2$ refractive indices, and likewise for $\epsilon 1>\epsilon 2[10,11]$. Applied optical waveguides with rectangular geometry are furthermost effortlessly assumed as variants of a hypothetical dielectric slab waveguide, as so-called planar waveguide. The planar waveguide is consisted of three different materials layers as well as dissimilar dielectric constant, which infinitely extending in a particular direction that parallel to the interface.

The total internal reflection causes the confinement of light in the middle later. This phenomenon only occurs if the dielectric index of the surrounding layers is larger than that of the middle layer [12]. Particularly, it is assumed that the slab waveguides are finite in the direction parallel to the interface. However, in the case of large interfaces typical size, as compared to layer depth, the model of slap waveguide is a perfect approximation [13]. It is not applicable for slab waveguide guided modes to be excited by the incident light from bottom or top interfaces. In contrast, light ought to be injected into the middle layer using a lens from the side. Otherwise, light to be coupled into the waveguide, coupling element can be utilized, for instance prism or grating coupler [14]. Such example of guided mode is a plane wave being reflected between the middle layer binary interfaces; this must occur at an incident angle between the light propagation direction and/or at perpendicular direction to the interface of the material which is higher than the critical angle. Subsequently, a critical angle relies on the material refraction index in which it can be varied depending on the light wavelength. Therefore, a guided mode is resulted from such propagation, this must occur at a discrete set of angles whereby the reflected wave of plane is not damagingly interfered with itself.

The electromagnetic waves are confined by the aforementioned structure which can only happen in one direction. Therefore, low probability of practical applications is expected. Structures that can be estimated as slab waveguides utilize, but occasionally happen in other devices [15]. As illustrated in Figure 2, TE modes with $\mathrm{Ez}=0$, the electric field is transverse to the propagation trend whilst the magnetic field is typical to the propagation trend. In contrast, in $\mathrm{TM}$ modes with $\mathrm{Hz}=0$, the magnetic field is transverse to the propagation trend while the electric field is normal to the propagation trend [16].

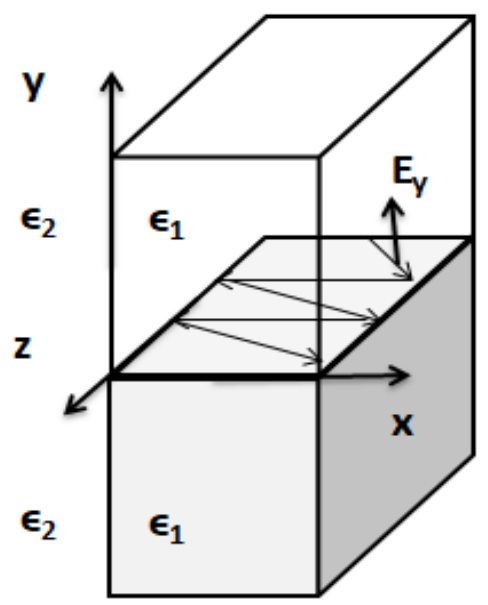

$2 a$

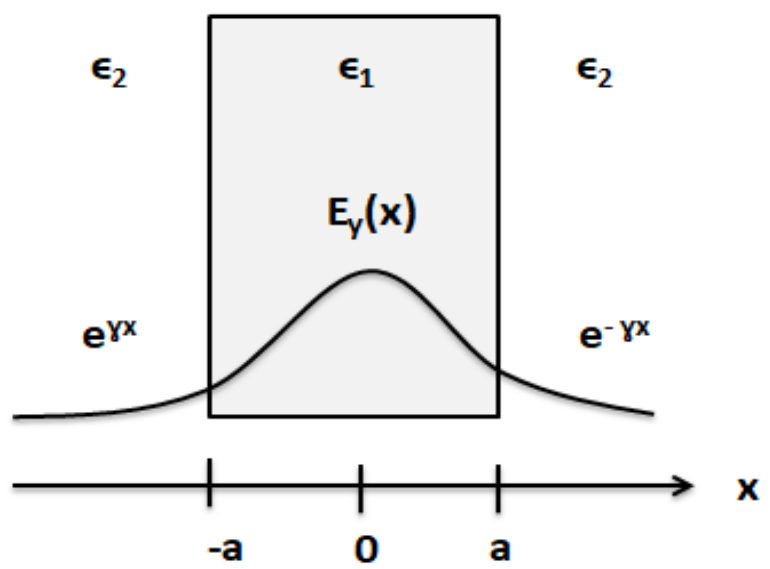

Figure 1. Dielectric planar waveguide 


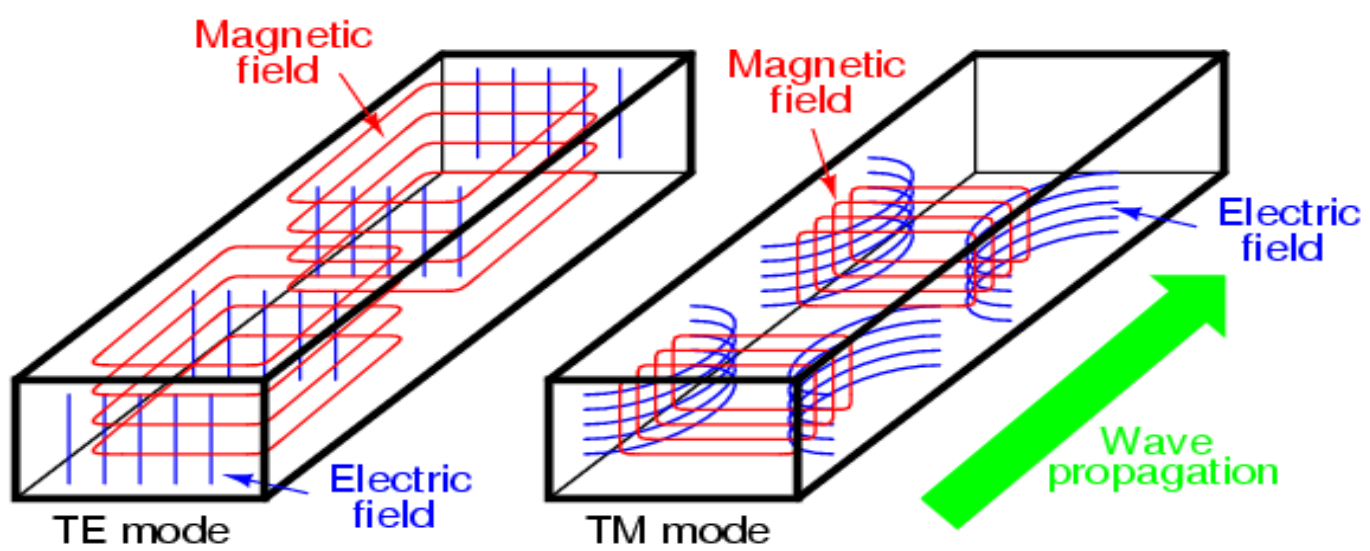

Figure 2. Waveguide transverse magnetic (TM) and transverse electric (TE) modes [17]

\section{$\mathrm{n}_{2}$ (cladding)}

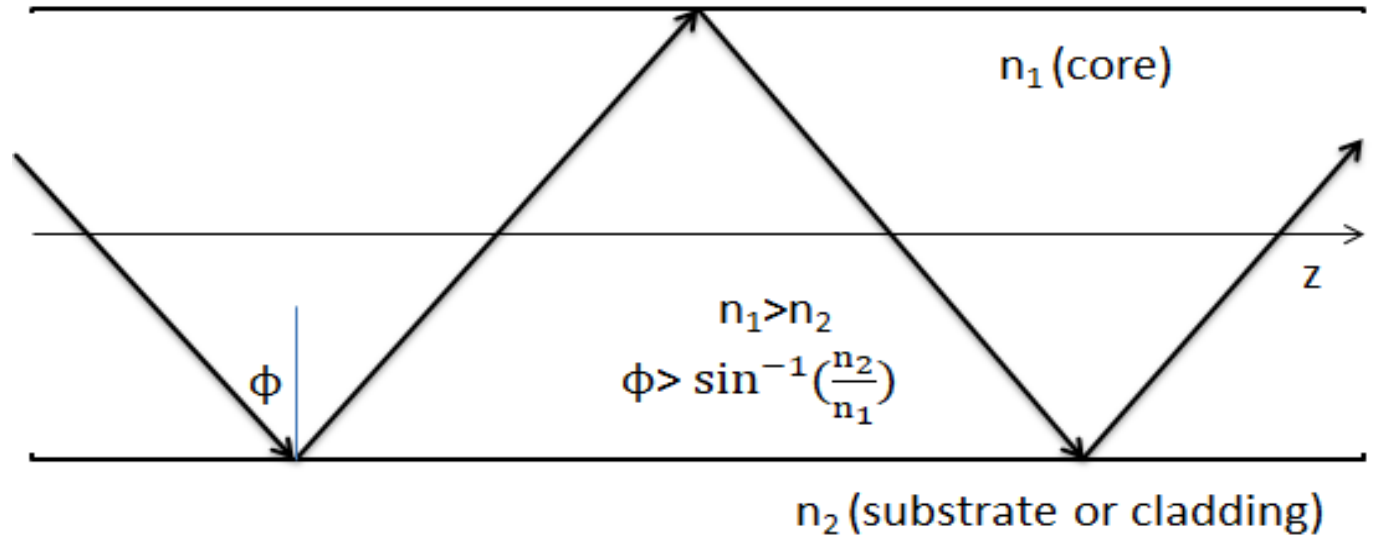

Figure 3. Propagation of light in waveguide by the principle of TIR

A planer waveguide, which is a simple structure of a normal waveguide, exhibits a total internal reflection (TIR) of infrared (IR) beam. The latter must exhibit an angle $\varnothing$ which is greater than the critical angle $\left(\theta_{c}=\sin ^{-1}\left(\frac{n_{2}}{n_{1}}\right)\right)$ in order to get TIR as shown in Figure 3.

\subsection{Parameters Value}

It is generally known that for guided mode $\mathrm{n}_{2}<\mathrm{n}_{\mathrm{eff}}<$ $\mathrm{n}_{1}$ by which $\mathrm{n}_{\text {eff }}$ is given as:

$$
\mathrm{n}_{\mathrm{eff}}=\frac{\beta}{\mathrm{k}_{\circ}}
$$

Where $\mathrm{n}_{2}$ is the cladding refractive index, while $\mathrm{n}_{\text {eff }}$ is the effective refractive index, $n_{1}$ is the core refractive index, $\beta$ is the propagation constant, and $k_{\circ}=\frac{2 \pi}{\lambda}$ is the wave number $\left(\mathrm{k} \circ \mathrm{n}_{2}<\beta<\mathrm{k} \circ \mathrm{n}_{1}\right)$

The boundary conditions for symmetric mode should be continuous at $x=\mp a$. The normalized frequency is given by the following equation:

$$
\mathrm{V}=\frac{2 \pi}{\lambda} * \frac{2 \mathrm{a}}{2} * \mathrm{NA}
$$

Where $\mathrm{V}$ is normalized frequency, $\mathrm{NA}$ is numerical aperture and it is given as:

$$
\mathrm{NA}=\sqrt{\mathrm{n}_{1}^{2}-\mathrm{n}_{2}^{2}}
$$

The cutoff frequency is then defined as:

$$
\mathrm{f}_{\mathrm{c}}=\frac{\mathrm{mc}}{4 \mathrm{aNA}}, \mathrm{m}=0,1,2,3,
$$

Where $\mathrm{c}$ is the speed of light in free space and $m$ is the number of modes.

The penetration depth, which is the distance scale corresponding to the waveguide penetration, determines the degradation within the waveguide. Through equation (5), the relation between the light wavelength of diffusion within the waveguide, the cladding refractive index, and the effective refractive index is described $[18,19]$.

$$
\begin{gathered}
d_{p}=\frac{1}{\mathrm{r}} \\
\text { OR } d_{p}=\frac{\lambda}{2 \pi \sqrt{\mathrm{n}_{\text {eff }}^{2}-\mathrm{n}_{2}^{2}}}
\end{gathered}
$$

Then, normalized propagation constant can be defined using equation (7), while the propagation of electromagnetic waves inside the waveguide (propagation angles) can be defined by equation (8) 


$$
\begin{gathered}
b=\frac{\mathrm{n}_{\text {eff }}-\mathrm{n}_{2}}{\mathrm{n}_{1}-\mathrm{n}_{2}} \\
\theta_{1}=\sin ^{-1}\left(\frac{\mathrm{n}_{\text {eff }}}{\mathrm{n}_{1}}\right)
\end{gathered}
$$

The critical angle is given by the following equation:

$$
\theta_{c}=\sin ^{-1}\left(\frac{n_{2}}{n_{1}}\right)
$$

\subsection{Explicit Eigen Solutions for TE and TM Modes}

The wave propagation inside a waveguide is represented by two functions which are symmetric and anti-symmetric. These functions, symmetric and anti-symmetric, are in turn represented by even and odd numbers of modes, respectively.

For even TE mode (symmetric function)

$$
\xi \tan (\xi)=\eta
$$

and, for odd TE mode (anti-symmetric function)

$$
-\xi \cot (\xi)=\eta
$$

Where $\xi=\mathrm{ka}$ and $\eta$ is given by equation (12)

$$
\eta=\sqrt{\mathrm{V}^{2}-\xi^{2}} \quad \text { (circles) }
$$

For even TM mode (symmetric function)

$$
\xi \tan (\xi)=\frac{n_{1}^{2}}{n_{2}^{2}} \eta
$$

And, for odd TM mode (anti-symmetric function)

$$
\begin{gathered}
-\xi \cot (\xi)=\frac{n_{1}^{2}}{n_{2}^{2}} \eta \\
\eta=\frac{n_{1}}{n_{2}} \sqrt{V^{2}-\xi^{2}} \quad \text { (ellipse) }
\end{gathered}
$$

\subsection{TE Mode Wave Equation}

The following equations are generated to describe TE mode, and the waveform inside the waveguide TE mode is found by solving these equations.

$$
\begin{aligned}
& \beta \mathrm{E}_{\mathrm{y}}=-\omega \mu \mathrm{H}_{\mathrm{x}} \\
& \frac{\partial \mathrm{Hz}}{\partial \mathrm{x}}+\mathrm{j} \beta \mathrm{H}_{\mathrm{x}}=-\mathrm{j} \omega \varepsilon \mathrm{E}_{\mathrm{y}} \\
& \frac{\partial \mathrm{E}_{\mathrm{y}}}{\partial \mathrm{x}}=-\mathrm{j} \omega \varepsilon \mathrm{H}_{\mathrm{z}} \\
& \frac{\partial^{2} E_{y}}{\partial x^{2}}+k^{2} E_{y}=0 \quad \text { for }|x|<a \\
& \text { Let } \mathrm{k}^{2}=\mathrm{k}_{\circ}^{2} \mathrm{n}_{1}^{2}-\beta^{2} \\
& \frac{\partial^{2} E_{y}}{\partial x^{2}}+\gamma^{2} E_{y}=0 \quad \text { for }|x|>a \\
& \text { Let } \gamma^{2}=\beta^{2}-k_{\circ}^{2} n_{2}^{2}
\end{aligned}
$$

Where $\mathrm{k}$ is Cutoff wave number, $\mathrm{\gamma}$ is attenuation coefficient.

\subsection{TM Mode Wave Equation}

The solution to the wave equation in TM mode is similar to that in TE mode, but the difference between them is the effect of the refractive index on the vector evidence in TM mode, since $\varepsilon=\varepsilon_{\circ} \mathrm{n}_{2}$

$$
\begin{gathered}
\beta H_{y}=\omega \varepsilon E_{x} \\
\frac{\partial H_{y}}{\partial x}=j \omega \varepsilon E_{z} \\
\frac{\partial E_{z}}{\partial x}+j \beta E_{x}=j \omega \mu H_{y}
\end{gathered}
$$

\subsection{Temperature Effect in Refractive Index of $\mathrm{ZnS}$}

The temperature has an effect on the material, it makes the electrons in the matter move faster. The refractive index of a substance changes with temperature change, and this is called the visual effect [20]. Through the equation (22), it is clearly understood that the relation between effective refractive index and temperature is proportional.

$$
\mathrm{n}_{\mathrm{T}}=\mathrm{n}_{\mathrm{R}}+(\mathrm{T}-\mathrm{R})\left(\frac{\mathrm{dn}}{\mathrm{dT}}\right)
$$

Where $\mathrm{R}$ is the room temperature, $\mathrm{T}$ is the temperature ranging from $0-460{ }^{\circ} \mathrm{C}, \mathrm{n}_{\mathrm{R}}$ and $\mathrm{n}_{\mathrm{T}}$ are effective refractive index at room temperature and ranged temperature, $\mathrm{dn} / \mathrm{dT}$ is the thermos optic constant which is $4.21 * 10^{-5}{ }^{\circ} \mathrm{C}$ for zinc sulfide.

\section{Calculation and Result}

The number of modes, calculated using equations (12) and (15), in both TE and TM are represented in Figure 4, while the waveguide core refractive index and thickness are tabulated in Table 1. As presented in the figure, both TE and TM modes exhibited an increased number of modes as the core thickness increases. In the mean while, it should be mentioned that TE and TM modes are presented in circular and ellipse shapes, respectively.

Table 1. Stratum of waveguide

\begin{tabular}{|c|c|c|}
\hline & Refractive index & Thickness \\
\hline Core & $\mathbf{n}_{\mathbf{1}}=2.32$ & $0.74 \mu \mathrm{m}, 1.48 \mu \mathrm{m}$ \\
& $\mathbf{n}_{\mathbf{2}}=2.22$ & $-2 \mu \mathrm{m}$ and $2.96 \mu \mathrm{m}$ \\
\hline Cladding & \multicolumn{2}{|c|}{} \\
\hline
\end{tabular}

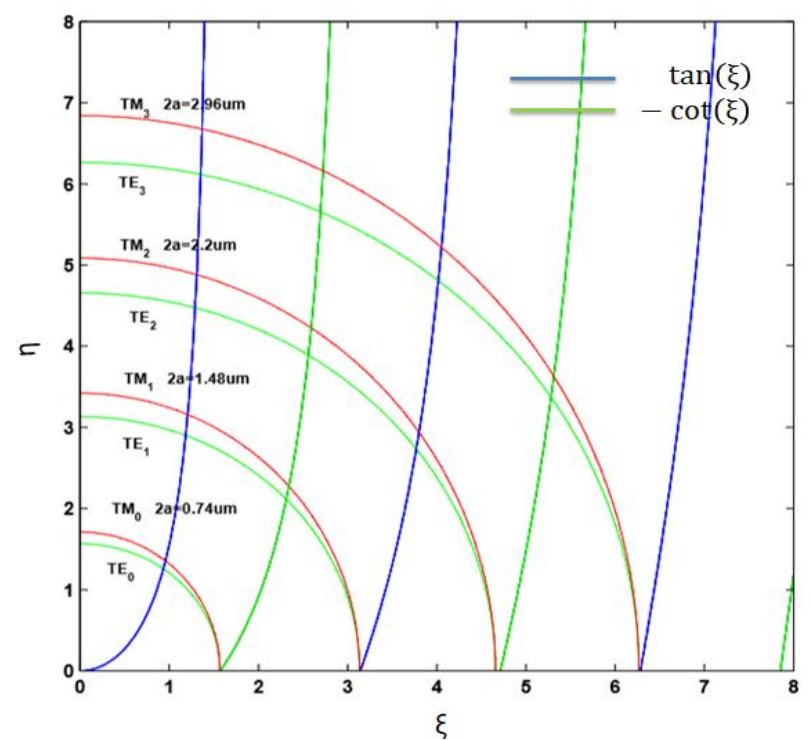

Figure 4. Number of modes (TE and TM) for different values of thickness in graphical solution for $\mathrm{n}_{1}=2.32$ and $\mathrm{n}_{2}=2.22$ 
Table 2. Parameters in waveguide when $\lambda=1 \mathrm{um}$ and $2 \mathrm{a}=2.96 \mu \mathrm{m}$

\begin{tabular}{|c|c|c|c|}
\hline & $\mathbf{n}_{\mathbf{f f f}}$ & $\mathbf{d}_{\mathbf{p}}$ & $\mathbf{\gamma}$ \\
\hline $\mathbf{T E}_{\mathbf{0}}$ & 2.3154 & 0.2419 & 4.1335 \\
\hline $\mathbf{T M}_{\mathbf{0}}$ & 2.3135 & 0.2445 & 4.0904 \\
\hline $\mathbf{T E}_{\mathbf{1}}$ & 2.3018 & 0.2617 & 3.8216 \\
\hline $\mathbf{T M}_{\mathbf{1}}$ & 2.2969 & 0.2700 & 3.7035 \\
\hline $\mathbf{T E}_{\mathbf{2}}$ & 2.2795 & 0.3077 & 3.2500 \\
\hline $\mathbf{T M}_{\mathbf{2}}$ & 2.2709 & 0.3328 & 3.0046 \\
\hline $\mathbf{T E}_{\mathbf{3}}$ & 2.2494 & 0.4393 & 2.2764 \\
\hline $\mathbf{T M}_{\mathbf{3}}$ & 2.2354 & 0.6079 & 1.6449 \\
\hline
\end{tabular}

As demonstrated in Table 2, the $\mathrm{n}_{\text {eff }}$ values were found to be higher in TE modes as compared to their identical in TM modes due to higher value of propagation constant in TE mode. The highest value of $\mathrm{n}_{\text {eff }}$ in $\mathrm{TE}_{3}$ and $\mathrm{TM}_{3}$ modes are 2.2494 and 2.2354 , respectively. In contrast to the $\mathrm{n}_{\text {eff }}$, the penetration depth demonstrated higher value in TM modes, this can be attributed to the fact that the relation between the $\mathrm{n}_{\mathrm{eff}}$ and penetration depth is inversely proportional (Equation 6). As for the attenuation coefficient, calculated results (in accordance with Equation 20) were found to demonstrate higher values in TE mode.

The wave form in both TE and TM modes using different core thickness $(0.74,1.48,2.2$ and $2.96 \mu \mathrm{m})$ are illustrated in Figure 5. Generally, it was noticed that the number of modes rises with the core thickness increment. Furthermore, a single symmetry mode was observed with core thickness of $0.74 \mu \mathrm{m}$ (Figure 5, a), while further increment in the core thickness resulted in multi symmetry and anti-symmetry modes (Figure 5, b, c, and d). Concurrently, a slight width difference of the wave was observed whereby TE mode demonstrated broader wave form than TM mode. This phenomenon is due the fact that values of the propagation constant in TE mode is higher than that in $\mathrm{TM}$ mode $\left(\beta_{\mathrm{TE}}>\beta_{\mathrm{TM}}\right)$ which in turn can be attributed to the effect of refractive index on TM mode. The propagation angles used in this study are depicted in Table 3. It is observed from the aforementioned tables that the propagation angles in TM modes exhibited lower values than TE modes with respect to the core thickness.

Table 3. The propagation angles $\theta_{1}$ when $\lambda=1 \mu \mathrm{m}$ for $\mathrm{m}=0,1,2,3$.

\begin{tabular}{|c|c|c|c|}
\hline \multirow{2}{*}{ Mode } & \multicolumn{3}{|c|}{ Propagation angle $\boldsymbol{\theta}_{\mathbf{1}}$} \\
\cline { 2 - 4 } & $\mathbf{2 a = 1 . 4 8} \boldsymbol{\mu m}$ & $\mathbf{2 a = 2 . 2} \boldsymbol{\mu m}$ & $\mathbf{2 a = 2 . 9 6} \boldsymbol{\mu m}$ \\
\hline TE0 & 1.4613 & 1.4904 & 1.5078 \\
\hline TM0 & 1.4510 & 1.4827 & 1.4958 \\
\hline TE1 & 1.3546 & 1.4106 & 1.4455 \\
\hline TM1 & 1.3344 & 1.3958 & 1.4296 \\
\hline TE2 & - & 1.3333 & 1.3837 \\
\hline TM2 & - & 1.3110 & 1.3647 \\
\hline TE3 & - & - & 1.3235 \\
\hline TM3 & - & - & 1.2999 \\
\hline
\end{tabular}
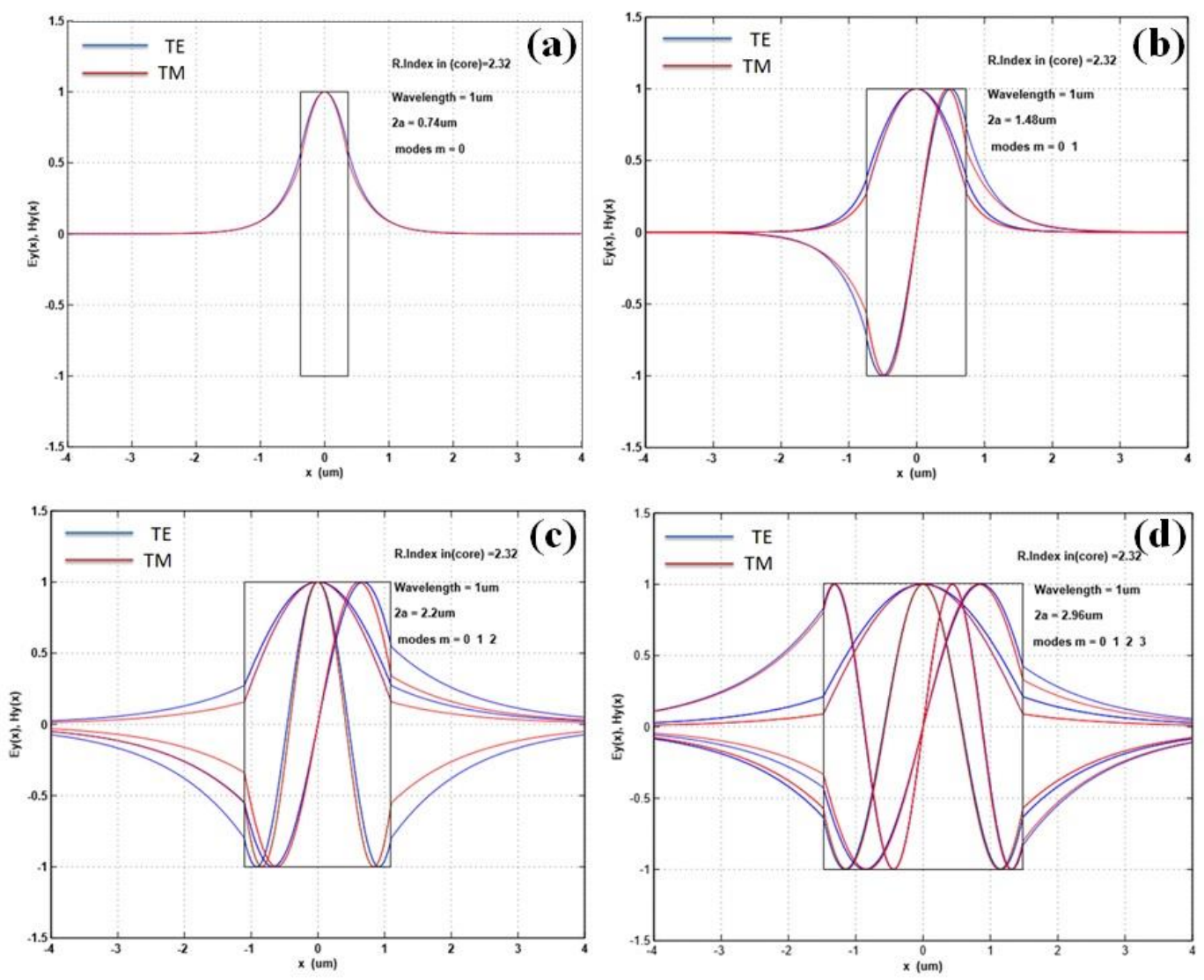

Figure 5. The difference in the number of modes with waveguide thickness in TE and TM modes where $E_{y}(x)$ for $T E$ and $H_{y}(X)$ for $T M(V / \mu m)$ 

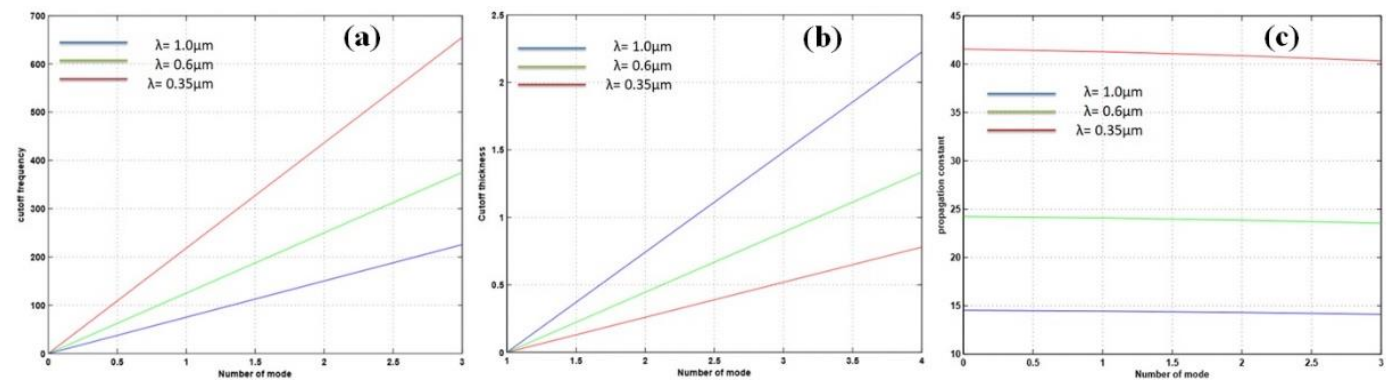

Figure 6. (a)The relationship between (Number of mode and cutoff frequency) (b)The relationship between (Number of mode and cutoff thickness) (c)The relationship between (Number of mode and propagation constant)

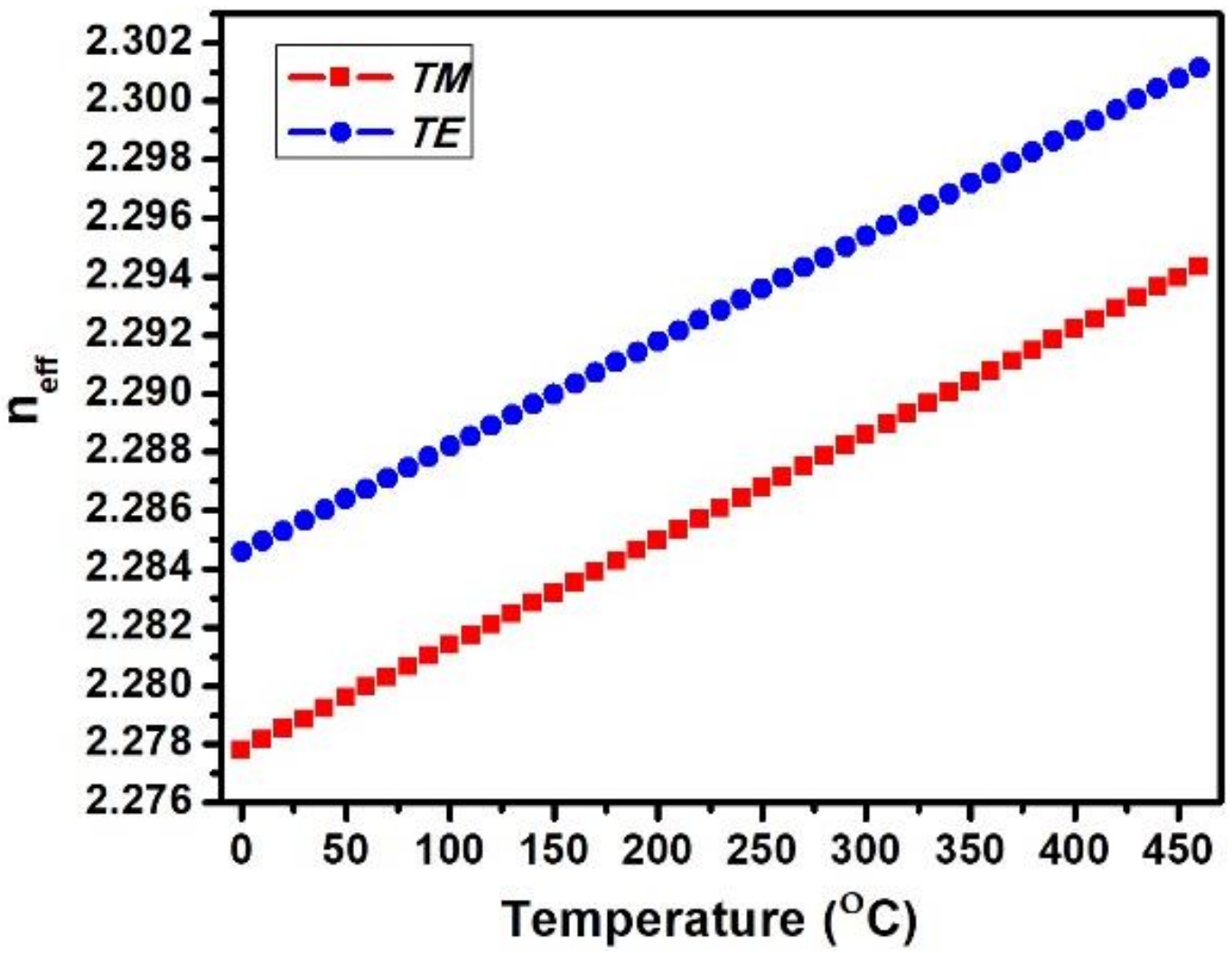

Figure 7. The relationship between temperature and effective refractive index

Figure 6(a) shows the relation between wavelength variation and cutoff frequency. It can be clearly perceived that an inversely proportional relation between the wavelength and cutoff frequency is found, whereby lower wavelength resulted in higher cutoff frequency with respect to the number of modes. In contrast, the cutoff thickness was found with higher values as the wavelength decreases (Figure 6, b). Continuously, in Figure 6 (c), it is perceived that increment in the wavelength value leaded to an increase in the values of propagation constant. Contrariwise, the pronounced propagation constant decreased with increment of the number of modes (Figure $6, c)$.

Figure 7 depicts the relation between $n_{\text {eff }}$ and temperature for both TE and TM modes. As demonstrated in the figure, an increase in the temperature from 0 to
$460{ }^{\circ} \mathrm{C}$ resulted in pronounced values of $n_{\text {eff. }}$ In the meanwhile, it is noteworthy to mention that TE mode exhibited advanced values as compared to TM mode.

\section{Conclusions}

The theoretical analysis of spread electromagnetic waves via $\mathrm{ZnS}$ waveguide was successfully reported. A detailed analysis of the waveguide parameters was demonstrated using both TE and TM modes, where by lower propagation angle values were noticed using TM mode. This singularity was found to be due to the interference of effective refractive index value. The penetration depth demonstrated values of 0.2419 and $0.2445 \mu \mathrm{m}$ for $\mathrm{TE}_{0}$ and $\mathrm{TM}_{0}$ modes at thickness of $2.69 \mu \mathrm{m}$, 
respectively. Subsequently, the effect of temperature on effective reflective index has been elaborated. This work represents a short pathway for the theoretical analysis concerning the electromagnetic waves propagating through $\mathrm{ZnS}$ planer waveguide with both modes (TE and TM) considered.

\section{REFERENCES}

[1] L. Ali, N.I. Fawaz, M.H. Suhail, Dielectric Waveguide for Composite Materials, Iraqi Journal of Physics, 9 (2011) $39-46$.

[2] B. Perry, P. Rabinowitz, M. Newstein, Wave propagation in media with focused gain, Physical Review A, 27 (1983) 1989.

[3] D.R. Lynch, K.D. Paulsen, Origin of vector parasites in numerical Maxwell solutions, IEEE Transactions on Microwave Theory and Techniques, 39 (1991) 383-394.

[4] I. Arifianto, M.R. Hadisiswoyo, A. Syahriar, Modelling Temperature Effect on (Transverse Electric) TE Mode Shape of Optical Silica Waveguide, (2019).

[5] N.I. Fawaz, A. Hameed, Cladding effects on Wave propagation in Dielectric Slab waveguide, in: Journal of Physics: Conference Series, IOP Publishing, 2019, pp. 012023.

[6] E. Thostenson, T.-W. Chou, Microwave processing: fundamentals and applications, Composites Part A: Applied Science and Manufacturing, 30 (1999) 1055-1071.

[7] N.Y. Gordeev, A.S. Payusov, Y.M. Shernyakov, S.A. Mintairov, N.A. Kalyuzhnyy, M.M. Kulagina, M.V. Maximov, Transverse single-mode edge-emitting lasers based on coupled waveguides, Optics letters, 40 (2015) 2150-2152.

[8] J. Zhou, M. Chen, R. Zhong, S. Liu, Analysis of TM and TE Modes in Eccentric Coaxial Lines Based on Bipolar
Coordinate System, Applied Computational Electromagnetics Society Journal, 30 (2015).

[9] D. Marcuse, Theory of dielectric optical waveguides, Elsevier, 2013.

[10] C.R. Pollock, M. Lipson, Integrated photonics, Springer, 2003.

[11] P.v. Tien, Light waves in thin films and integrated optics, Applied optics, 10 (1971) 2395-2413.

[12] F. Zangeneh-Nejad, R. Fleury, Acoustic analogues of high-index optical waveguide devices, Scientific reports, 8 (2018) 10401.

[13] L. La Spada, S. Haq, Y. Hao, Modeling and design for electromagnetic surface wave devices, Radio Science, 52 (2017) 1049-1057.

[14] X. Chen, C. Li, H.K. Tsang, Fabrication-tolerant waveguide chirped grating coupler for coupling to a perfectly vertical optical fiber, IEEE Photonics Technology Letters, 20 (2008) 1914-1916.

[15] V.A.G. Rivera, Ion exchange to fabrication of waveguides for optical telecommunication, in: Ion Exchange Technology I, Springer, 2012, pp. 467-490.

[16] TEST \& MEASUREMENT TIPS, Basics of TEM, TE, and TM propagation, in, 2015, September 11.

[17] E. Done, TE \& TM Modes, in, 2020, March 14.

[18] N.T. Beneitez, J. Missinne, J. Schleipen, J. Orsel, M.W. Prins, G. Van Steenberge, Polymer slab waveguides for the optical detection of nanoparticles in evanescent field based biosensors, in: Nanoscale Imaging, Sensing, and Actuation for Biomedical Applications XI, International Society for Optics and Photonics, 2014, pp. 89540Q.

[19] N.Z. Zhao, I.A. Williamson, Z. Zhao, S. Boutami, S. Fan, Penetration depth reduction with plasmonic metafilms, ACS Photonics, 6 (2019) 2049-2055.

[20] M. Huang, X. Yan, Thermal-stress effects on the temperature sensitivity of optical waveguides, JOSA B, 20 (2003) 1326-1333. 